\title{
Genetic loci for serum magnesium among African-Americans and gene-environment interaction at MUC1 and TRPM6 in European- Americans: the Atherosclerosis Risk in Communities (ARIC) study
}

Adrienne Tin ${ }^{1 *}$, Anna Köttgen ${ }^{1,2}$, Aaron R Folsom ${ }^{3}$, Nisa M Maruthur ${ }^{4}$, Salman M Tajuddin ${ }^{5}$, Mike A Nalls ${ }^{5}$, Michele K Evans ${ }^{5}$, Alan B Zonderman ${ }^{5}$, Christopher A Friedrich ${ }^{6}$, Eric Boerwinkle”, Josef Coresh ${ }^{7}$ and Wen Hong Linda Kao ${ }^{{ }^{\wedge}}$

\begin{abstract}
Background: Low serum magnesium levels have been associated with multiple chronic diseases. The regulation of serum magnesium homeostasis is not well understood. A previous genome-wide association study (GWAS) of European ancestry (EA) populations identified nine loci for serum magnesium. No such study has been conducted in African-Americans, nor has there been an evaluation of the interaction of magnesium-associated SNPs with environmental factors. The goals of this study were to identify genetic loci associated with serum magnesium in an African-American (AA) population using both genome-wide and candidate region interrogation approaches and to evaluate gene-environment interaction for the magnesium-associated variants in both EA and AA populations. We conducted a GWAS of serum magnesium in 2737 AA participants of the Atherosclerosis Risk in Communities (ARIC) Study and interrogated the regions of the nine published candidate loci in these results. Literature search identified the influence of progesterone on MUC1 expression and insulin on TRPM6 expression.
\end{abstract}

Results: The GWAS approach in African-American participants identified a locus near MUC1 as genome-wide significant ( $r$ 2974937, beta $=-0.013, p=6.1 \times 10^{-9}$ ). The candidate region interrogation approach identified two of the nine loci previously discovered in EA populations as containing SNPs that were significantly associated in African-American participants (SHROOM3 and TRPM6). The index variants at these three loci together explained $2.8 \%$ of the variance in serum magnesium concentration in ARIC African-American participants. On the test of gene-environment interaction in ARIC EA participants, the index variant at MUC1 had 2.5 times stronger association in postmenopausal women with progestin use (beta $=-0.028, p=7.3 \times 10^{-5}$ ) than in those without any hormone use (beta $=-0.011, p=7.0 \times 10^{-8}$, $p$ for interaction 0.03). At TRPM6, the index variant had 1.6 times stronger association in those with lower fasting insulin levels (<80pmol/L: beta $=-0.013, p=1.6 \times 10^{-7} ; \geq 80 \mathrm{pmol} / \mathrm{L}$ : beta $=-0.008, p=1.8 \times 10^{-2}, \mathrm{p}$ for interaction 0.03 ).

Conclusions: We identified three loci that explained $2.8 \%$ of the variance in serum magnesium concentration in ARIC African-American participants. Following-up on functional studies of gene expression identified gene-environment interactions between progestin use and MUC1 and between insulin and TRPM6 on serum magnesium concentration in ARIC European-American participants. These results extend our understanding of the metabolism of serum magnesium.

Keywords: Gene-environment interaction, Serum magnesium, MUC1, TRPM6

\footnotetext{
*Correspondence: atin1@jhu.edu

Deceased

'Johns Hopkins Bloomberg School of Public Health, Baltimore, MD, USA

Full list of author information is available at the end of the article
} 


\section{Background}

Low serum magnesium levels have been associated with multiple common chronic diseases, including cardiovascular diseases [1], hypertension [2], diabetes [3], and chronic kidney disease [4]. The regulation of serum magnesium hemostasis is not well understood. The heritability of serum magnesium is estimated to be $27 \%$ [5], suggesting genetic association studies of serum magnesium can improve our understanding of serum magnesium metabolism. A genome-wide association study (GWAS) in populations of European ancestry (EA) previously identified nine loci associated with serum magnesium levels [6], and these loci explained $<2 \%$ of serum magnesium inter-individual variation [6]. African-Americans (AA) are known to have lower serum magnesium levels [7], and no results from GWAS in AA populations have been reported. It is known that associated variants may be ancestry-specific [8]. Gene-environment interactions can also modify genetic effects. The investigation of genetic associations across ethnic groups and the identification of geneenvironment interactions have the potential to increase our understanding of the regulation of serum magnesium levels. The goals of the present study were to 1) identify genomic loci associated with serum magnesium in AA individuals using both genome-wide and candidate region interrogation approaches and 2) follow up on the GWAS findings from EA populations, to identify environmental factors that may modify the association between genetic loci and serum magnesium in both EA and AA populations.

We conducted a GWAS of serum magnesium in 2737 AA participants of the Atherosclerosis Risk in Communities (ARIC) Study and interrogated the regions of the nine published candidate loci from EA populations in the AA GWAS results. Index variants at loci that reached suggestive significance in AA participants of the ARIC study were put forward for replication in the Healthy Aging in Neighborhoods of Diversity across the Life Span (HANDLS) study. Geneenvironment interaction analyses were conducted in the ARIC study for MUC1 and TRPM6 based on the results of literature search, which identified the influence of progesterone on MUC1 expression and insulin on TRPM6 expression.

\section{Results}

\section{Study population characteristics}

In the ARIC study, the sample sizes were 2,737 for AA participants and 8,926 for EA participants with mean serum magnesium levels of $0.79 \mathrm{mmol} / \mathrm{L}$ and $0.83 \mathrm{mmol} / \mathrm{L}$ respectively (Table 1). The HANDLS study included 942 AA participants with mean serum magnesium levels of $0.77 \mathrm{mmol} / \mathrm{L}$.

\section{Serum magnesium loci in ARIC African-American participants}

The GWAS in AA participants identified 17 SNPs near or at MUC1 as genome-wide significant. The index SNP in ARIC AA participants was rs2974937 at THBS3, $2.7 \mathrm{~kb}$ upstream of $M U C 1$, (beta $=-0.013$, frequency $=0.32$, $p=6.1 \times 10^{-9}$, Table 2). The index SNP previously identified in EA populations, rs4072037, was also genomewide significant (beta $=-0.014 \mathrm{mmol} / \mathrm{L}$, frequency = $0.32, p=2.2 \times 10^{-8}$, Additional file 1: Table S1). The two index SNPs, rs4072037 and rs2974937, were in high linkage disequilibrium $\left(r^{2}>0.7\right.$ and $D^{\prime}>0.9$ in ARIC EA and AA participants and the 1000 Genomes Phase 1 data, Additional file 1: Table S2). Fig. 1 presents the plot of $-\log _{10}(P$-values $)$ by genomic position of the GWAS of serum magnesium in ARIC AA participants, and Additional file 1: Figure S1 and S2 present the quantile-quantile plot of the GWAS results and regional association plots of the MUC1 locus, respectively.

The index SNPs at eight other loci identified in EA populations did not have significant association (meta-analysis $p>0.01$ ) with serum magnesium in AA participants of the ARIC and HANDLS studies (Additional file 1: Table S1). In the GWAS of ARIC AA participants, SNPs at five other loci showed suggestive significance (MAF $\geq 5 \%$ and $p$ value $<10^{-6}$, Additional file 1 : Table S3). The index SNPs at these loci were not significantly associated with serum magnesium in the HANDLS study. The imputation quality of these index SNPs ranged from 0.58 to 0.97 in AA participants of the ARIC and HANDLS studies.

On the candidate region interrogation, the AA-specific index SNPs at five loci previously identified in EA populations (MUC1, MDS1, SHROOM3, TRPM6, and PRMT7) met the region-specific significant threshold (Table 2). The index SNPs at three of these five loci were replicated in the HANDLS study (MUC1, SHROOM3, and TRPM6). The AA-specific index SNPs at these three loci had imputation quality $\geq 0.87$ in the AA participants of the ARIC and HANDLS studies. In addition, at MUC1 and TRPM6, at least one genotyped SNP in ARIC AA participants was associated with serum magnesium below the regionspecific threshold (Additional file 1: Table S4). Additional file 1: Figs. S2, S3, S4 present the regional association plots from ARIC AA participants for these three loci. Enhancement enrichment tests using HaploReg showed all three loci were enriched for enhancers in one or more cell lines (Additional file 1: Table S5).

\section{Effect modification between the index SNP at MUC1 and progestin use}

The analysis for effect modification between MUC1 and progestin use was conducted in EA participants only, because in the ARIC AA participants only 24 postmenopausal 
Table 1 Study Participant Characteristics in the ARIC and HANDLS Studies

\begin{tabular}{llll}
\hline & & ARIC study & HANDLS study \\
\hline N & European-Americans & African-Americans & African-Americans \\
Age, mean (SD) & 8926 & 2737 & 942 \\
Female, \% (n) & $54.3(5.7)$ & $53.4(5.8)$ & $48.3(9.0)$ \\
Prevalent diabetes, \% (n) & $53.0(4728)$ & $62.6(1714)$ & $55.9(527)$ \\
Diuretic use, \% (n) & $8.4(749)$ & $18.7(513)$ & $32.2(303)$ \\
Estimated glomerula filtration rate $\left(\mathrm{mL} / \mathrm{min} / 1.73 \mathrm{~m}^{2}\right)$, mean (SD) & $14.5(1297)$ & $28.8(788)$ & $13.6(128)$ \\
Serum Mg (mmol/L), mean (SD) & $93.3(12.7)$ & $103.8(18.0)$ & $96.3(24.1)$ \\
\hline
\end{tabular}

Abbreviation: SD, standard deviation

women reported the use of progestin. The most likely genotype of the index SNP at MUC1 in EA populations, rs4072037, was independent of progestin use (Chi-square $p=0.21$ ). In women with progestin use, the effect size of rs4072037 was more than two-fold larger (beta $=$ $-0.028 \mathrm{mmol} / \mathrm{L}, p=7.3 \times 10^{-5}$ ) than in other groups without progestin use ( $\mathrm{p}$ for interaction $=0.01$ to 0.05 , Table 3 ). The phenotypic variance explained by rs4072037 in women with progestin use was $6.5 \%$ versus $\leq 1.2 \%$ for all other groups.

\section{Effect modification between the index SNPs at TRPM6 and fasting insulin}

At TRPM6, the index SNP identified in EA populations [6], rs11144134, had very low frequency in ARIC AA participants $(\mathrm{MAF}=2 \%$ ), and the index SNP in AA participants, rs113607577 (Table 2), was monomorphic in ARIC EA participants. Thus, the analysis for the effect modification between TRPM6 and fasting insulin levels was conducted in EA and AA participants using the index SNP within the respective populations. The most likely genotype of the index SNPs in both cohorts was independent of insulin levels (Chi-square $p=0.77$ in EA participants and 0.49 in AA participants).

In ARIC EA participants, the association between rs11144134 and serum magnesium levels was stronger in individuals with lower fasting insulin levels $(<80 \mathrm{mmol} / \mathrm{L}$, beta $\left.=-0.013, p=1.6 \times 10^{-7}\right)$ than in those with higher fasting insulin levels $(\geq 80 \mathrm{mmol} / \mathrm{L}$, beta $=-0.008, p=0.02$, $\mathrm{p}$ for interaction $=0.03$, Table 4). Similarly, in ARIC AA participants, the association between rs113607577 and serum magnesium levels was also stronger in individuals with lower fasting insulin levels $(<80 \mathrm{mmol} / \mathrm{L}$, beta $=$ $-0.026, p=3.5 \times 10^{-6}$ ) than in those with higher fasting insulin levels $\left(\geq 80 \mathrm{mmol} / \mathrm{L}\right.$, beta $=-0.014, p=9.0 \times 10^{-3}$, $\mathrm{p}$ for interaction $=0.24)$.

Two nonsynonymous SNPs at TRPM6 were reported to impair the response of TRPM6 to higher insulin levels (rs2274924 [K1584E] and rs3750425 [V1393I]) [9]. These two SNPs were in moderate linkage disequilibrium with the GWAS index SNPs at this locus (D' between 0.44 to
0.74 in ARIC EA and AA participants, Additional file 1: Table S6). In ARIC EA participants, the association between these two nonsynonymous SNP with serum magnesium appeared to be independent of rs11144134, the GWAS index SNP in EA populations. In ARIC AA participants, these two nonsynonymous SNPs were not associated with serum magnesium levels (Additional file 1: Table S7).

\section{Discussion \\ Main findings}

Three of nine loci associated with serum magnesium previously discovered in EA populations were found to be associated in AA populations (MUC1, TRPM6, and SHROOM3). MUC1 was genome-wide significant in ARIC AA participants. Together the index SNPs at these three loci explained $2.8 \%$ of serum magnesium variation in ARIC AA participants. In addition, we showed that in ARIC EA participants, the associations of serum magnesium with MUC1 and TRPM6 were modified by progestin use and insulin levels respectively, extending the findings of functional experiments on gene expression to genetic association studies at the population level.

\section{In the context of the literature}

The effect modification between the index SNP at $M U C 1$ and progestin use on serum magnesium levels is consistent with the known gene function of $M U C 1$ and supports $M U C 1$ as the causal gene at this locus. $M U C 1$ encodes a transmembrane mucin that forms part of the mucosal barrier in the intestine [10] and is also expressed in the kidney [11]. Higher progesterone levels increase the expression of MUC1 in endometrial cells through increased promoter activity $[12,13]$. The index SNPs in ARIC EA and AA participants were $6.7 \mathrm{~kb}$ apart and in high linkage disequilibrium, suggesting the two populations may share the same causal variant(s). The index SNP reported in EA populations (rs4072037), at $<1 \mathrm{~kb}$ upstream of the transcription start site, is in a regulatory region [14] and in moderate linkage disequilibrium with an expression tag SNP (rs4971093) of 
Table 2 Association between Loci Identified in Populations of European Ancestry and Serum Magnesium in African-American Populations

\begin{tabular}{|c|c|c|c|c|c|c|c|c|c|c|c|c|c|c|c|c|}
\hline \multirow[b]{2}{*}{ Locus } & \multirow[b]{2}{*}{$\begin{array}{l}\text { Index SNP in ARIC } \\
\text { AA participants }\end{array}$} & \multirow[b]{2}{*}{ Chr } & \multirow[b]{2}{*}{ Position } & \multirow[b]{2}{*}{$\begin{array}{l}\text { Coded/Non- } \\
\text { coded allele }\end{array}$} & \multicolumn{5}{|c|}{ ARIC study African-American } & \multicolumn{4}{|c|}{ HANDLS study African-American } & \multicolumn{3}{|c|}{ Meta-analysis } \\
\hline & & & & & $\begin{array}{l}\text { Coded } \\
\text { allele freq. }\end{array}$ & Beta & SE & P-Value & $\begin{array}{l}\text { Imput. } \\
\text { qual. }\end{array}$ & $\begin{array}{l}\text { Coded } \\
\text { allele freq. }\end{array}$ & Beta & P-Value & $\begin{array}{l}\text { Imput. } \\
\text { qual. }\end{array}$ & Beta & P-Value & $\begin{array}{l}\text { P-Value threshold } \\
\text { in region }\end{array}$ \\
\hline MUC1 & rs2974937 & 1 & 155168849 & $C / T$ & 0.32 & -0.013 & 0.002 & $6.1 \mathrm{E}-09$ & 0.95 & 0.35 & -0.007 & 3.5E-02 & 0.99 & -0.012 & $2.1 \mathrm{E}-11$ & $8.9 \mathrm{E}-04$ \\
\hline MDS1 & rs74516251 & 3 & 168830777 & $A / G$ & 0.11 & -0.013 & 0.003 & $1.6 \mathrm{E}-04$ & 0.99 & 0.11 & -0.005 & $3.3 \mathrm{E}-01$ & 0.97 & -0.011 & 2.0E-05 & $4.9 \mathrm{E}-04$ \\
\hline SHROOM3 & rs9993810 & 4 & 77614640 & $A / G$ & 0.47 & -0.008 & 0.002 & $1.8 \mathrm{E}-04$ & 0.92 & 0.48 & -0.008 & $3.5 \mathrm{E}-02$ & 0.93 & -0.008 & $6.1 \mathrm{E}-06$ & 7.4E-04 \\
\hline TRPM6 & rs113607577 & 9 & 77455459 & $\mathrm{G} / \mathrm{C}$ & 0.90 & -0.019 & 0.004 & 1.1E-07 & 0.90 & 0.89 & -0.012 & 4.7E-02 & 0.87 & -0.017 & 4.6E-07 & 1.5E-03 \\
\hline DCDC51 & rs7947033 & 11 & 30795987 & $C / T$ & 0.10 & -0.011 & 0.004 & $5.4 \mathrm{E}-03$ & 0.74 & 0.08 & -0.001 & $8.3 \mathrm{E}-01$ & 0.91 & -0.008 & $1.4 \mathrm{E}-02$ & $1.0 \mathrm{E}-03$ \\
\hline ATP2B 11 & rs36089488 & 12 & 90118300 & $\mathrm{~T} / \mathrm{C}$ & 0.95 & -0.015 & 0.005 & $5.0 \mathrm{E}-03$ & 0.78 & 0.94 & -0.013 & $1.0 \mathrm{E}-01$ & 0.85 & -0.015 & $6.5 \mathrm{E}-04$ & $9.8 \mathrm{E}-04$ \\
\hline HOXD91 & rs6745764 & 2 & 176974122 & $\mathrm{G} / \mathrm{A}$ & 0.67 & -0.008 & 0.002 & $9.5 \mathrm{E}-04$ & 0.82 & 0.69 & -0.005 & 1.9E-01 & 0.90 & -0.007 & 3.1E-05 & $8.5 \mathrm{E}-04$ \\
\hline LUZP2 & rs11027876 & 11 & 24412973 & $\mathrm{G} / \mathrm{C}$ & 0.18 & -0.009 & 0.003 & $1.3 \mathrm{E}-03$ & 0.86 & 0.18 & -0.001 & $8.3 \mathrm{E}-01$ & 0.89 & -0.007 & $8.1 \mathrm{E}-03$ & $6.4 \mathrm{E}-04$ \\
\hline PRMT7 & rs55857142 & 16 & 68236741 & T/A & 0.07 & -0.018 & 0.004 & 2.7E-05 & 0.95 & 0.07 & 0.006 & $3.5 \mathrm{E}-01$ & 0.97 & -0.012 & 7.1E-04 & $9.4 \mathrm{E}-04$ \\
\hline
\end{tabular}

Betas are in $\mathrm{mmol} / \mathrm{L}$

Abbreviation: freq., frequency; $\mathrm{Chr}$, chromosome; SNP, single nucleotide polymorphism; SE, standard error; imput. qual., imputation quality

Bold indicates significant association in African-American populations based on genome-wide or region-specific $p$-value threshold and replication in the HANDLS study. The $\mathrm{I}^{2}$, an indicator for heterogeneity between study, was $45 \%$ for the index SNP at MUC1, 0 for SHROOM3, and $8 \%$ for TRPM6 


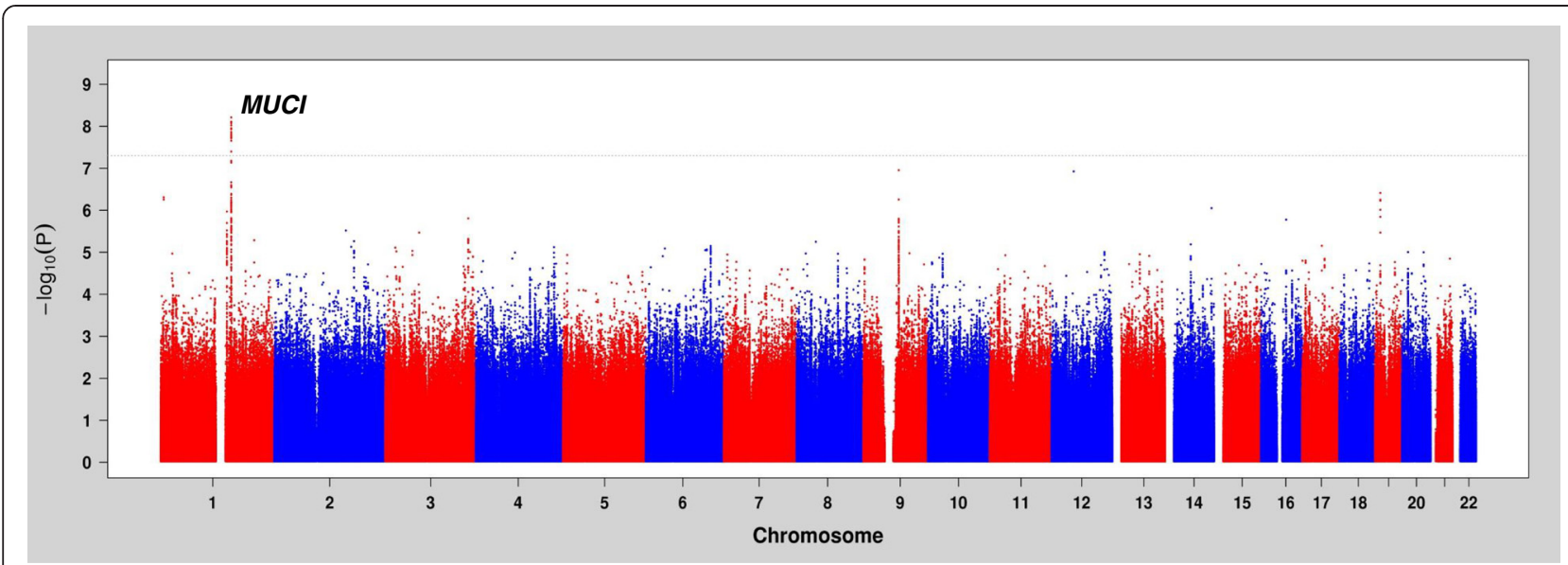

Fig. 1 A plot of $-\log _{10}(P$-values) by genomic position of the GWAS results of serum magnesium in ARIC African-American participants

MUC1 [15]. The minor allele of the index SNP was associated with decreased serum magnesium levels with significantly stronger association in women with progestin use. A potential explanation could be that the role of $M U C 1$ as part of the intestinal mucosal barrier affects the paracellular absorption of magnesium, and that genetic effects on $M U C 1$ become more apparent when there is increased $M U C 1$ expression.

In contrast, TRPM6 is a magnesium transporter involved in active magnesium absorption in the intestine and reabsorption in the distal convoluted tubule of the kidney [16, 17]. Higher insulin levels increase the expression of TRPM6 mediated by insulin receptors [9]. Although the index SNPs at this locus in EA and AA participants were not in high linkage disequilibrium and more than $25 \mathrm{~kb}$ apart, the major alleles of both index SNPs were associated with lower serum magnesium levels. This association was attenuated in individuals with higher insulin levels. If higher insulin increases the transcription of TRPM6 and leads to increase magnesium absorption, a potential explanation could be that the effect of the causal variant(s) at TRPM6 is less apparent when there are more TRPM6 receptors to transport magnesium. Since magnesium transport in kidney and intestine also occurs through additional routes and there are multiple ways by which higher insulin could influence serum magnesium levels, more complex explanations are also conceivable. The GWAS index SNPs in ARIC EA and AA participants were largely independent of the two nonsynonymous SNP found to influence TRPM6 transcription levels [9]. This suggests that multiple functional variants associated with serum magnesium exist at TRPM6.

\section{Strengths and limitations}

The strengths of this study include sufficient sample size for identifying the associations of serum magnesium loci previously discovered in EA populations in ARIC AA participants using a candidate region interrogation approach. We also have adequate sample size to assess gene-environment interaction in ARIC EA participants. Some limitations warrant mentioned. The suggestive significant serum magnesium loci identified in ARIC AA participants did not replicate in the HANDLS study, which could be due to a number of reasons, including limited power, moderate imputation quality, or type I error in the discovery sample. The association of the AA-specific index SNP at SHROOM3 with serum magnesium has not been verified by genotyped SNPs, although the association was replicated in the HANDLS study. The interaction between $M U C 1$ and progestin use in ARIC EA participants was not assessed in another study. The interaction between TRPM6 and insulin levels was significant in ARIC EA participants but not in ARIC AA participants. However, the direction of association was consistent

Table 3 Association Between rs4072037 C allele at MUC1 and Serum Magnesium by Gender and Progestin Use Status in ARIC European Americans

\begin{tabular}{|c|c|c|c|c|c|c|}
\hline & $\mathrm{N}$ & Beta (mmol/L) & SE & $P$-Value & Variance explained & $P$-Value for interaction \\
\hline Male & 4198 & -0.009 & 0.002 & $7.5 \mathrm{E}-09$ & $0.8 \%$ & 0.02 \\
\hline Female, premenopausal & 1428 & -0.012 & 0.003 & $2.8 \mathrm{E}-05$ & $1.2 \%$ & 0.05 \\
\hline Female, postmenopausal with no hormone use & 2495 & -0.011 & 0.002 & 7.0E-08 & $1.1 \%$ & 0.03 \\
\hline Female, postmenopausal with non-progestin hormone use & 574 & -0.005 & 0.004 & $2.4 \mathrm{E}-01$ & $0.2 \%$ & 0.01 \\
\hline Female, postmenopausal with progestin use & 231 & -0.028 & 0.007 & 7.3E-05 & $6.5 \%$ & Reference \\
\hline
\end{tabular}


Table 4 Association between Index SNPS at TRPM6 and Serum Magnesium by Fasting Insulin Levels in ARIC European and African Americans

\begin{tabular}{|c|c|c|c|c|c|c|}
\hline & $\mathrm{N}$ & Beta $(\mathrm{mmol} / \mathrm{L})$ & SE & $P$-Value & Variance explained & P for interaction \\
\hline \multicolumn{7}{|c|}{ European-Americans (rs11144134 T allele, frequency 0.92) } \\
\hline Fasting insulin $<80 \mathrm{pmol} / \mathrm{L}$ & 5937 & -0.013 & 0.002 & $1.6 \mathrm{E}-07$ & $0.44 \%$ & 0.03 \\
\hline Fasting insulin $\geq 80 \mathrm{pmol} / \mathrm{L}$ & 2752 & -0.008 & 0.003 & $1.8 \mathrm{E}-02$ & $0.19 \%$ & \\
\hline \multicolumn{7}{|c|}{ African-Americans (rs113607577 G allele, frequency 0.90 ) } \\
\hline Fasting insulin $<80 \mathrm{pmol} / \mathrm{L}$ & 1186 & -0.026 & 0.006 & 3.5E-06 & $1.55 \%$ & 0.24 \\
\hline Fasting insulin $\geq 80 \mathrm{pmol} / \mathrm{L}$ & 1302 & -0.014 & 0.005 & $9.0 \mathrm{E}-03$ & $0.58 \%$ & \\
\hline
\end{tabular}

between ARIC EA and AA participants. Post-hoc power analysis suggests the lack of significant association on the interaction between TRPM6 and insulin levels in ARIC AA participants is likely due to low power.

\section{Conclusion}

In summary, we identified three loci (MUC1, SHROOM3, and TRPM6) as associated with serum magnesium in African-Americans. In addition, we showed that two serum magnesium associated loci, MUC1 and TRPM6, had significant effect modification with progestin use and insulin levels, respectively, in European Americans. These findings extend previous research on magnesium metabolism and demonstrate gene-environment interaction can be identified systematically by following up on findings from gene expression functional experiments.

\section{Methods}

\section{Study population}

The ARIC Study is a community-based prospective observational study of individuals between the ages of 45 and 64 years. Participants were drawn from a probability-based sample from 4 US communities (Forsyth County, NC; Jackson, MS; suburban Minneapolis, MN; and Washington County, MD). The baseline visit occurred between 1987 and 1989. Details of the ARIC cohort have been published elsewhere [18].

Of the total 15,792 participants in the ARIC study, 9,044 EA and 2,874 AA participants had genotypes that met quality control criteria for GWAS (Additional file 1: Supplementary Methods section). Participants with estimated glomerular filtration rate (eGFR) $<30 \mathrm{~mL} / \mathrm{min} /$ $1.73 \mathrm{~m}^{2}$ (7 in EA, 97 in AA), missing diabetes status (12 in EA, 10 in AA), missing serum magnesium or having serum magnesium outside of 3 standard deviations within the specific population (99 in EA, 30 in AA) were not included in the analysis. As a result, the analyzed sample in the ARIC study included 8,926 EA participants and 2,737 AA participants. The threshold of $30 \mathrm{~mL} / \mathrm{min} / 1.73 \mathrm{~m}^{2}$ for eGFR was applied because the kidney's ability to maintain serum magnesium levels within normal range deteriorates substantially when
eGFR falls below this level [19]. Among EA participants, mean serum magnesium levels were slightly higher in those included in the analysis $(0.83 \mathrm{mmol} / \mathrm{L})$ than in those excluded $(0.82 \mathrm{mmol} / \mathrm{L}, p=0.003)$. Among AA participants, mean serum magnesium levels were similar between those included and excluded in the analysis (0.79 $\mathrm{mmol} / \mathrm{L}$ in both).

The HANDLS study, the replication cohort for serum magnesium association in African-Americans, is a community-based, longitudinal study of socioeconomically diverse AA and EA individuals in Baltimore [20]. A total of 942 AA participants with imputed genotype and data in serum magnesium and covariates were included in the replication analysis.

\section{Serum magnesium and other measures}

In the ARIC study, serum magnesium was measured using the metallochromic dye, Calmagite, based on the procedure of Gindler and Heth [21]. Prevalent diabetes mellitus was defined as a having a fasting glucose level at least $126 \mathrm{mg} / \mathrm{dL}$, nonfasting glucose level at least $200 \mathrm{mg} / \mathrm{dL}$, self-reported use of diabetes medication or self-reported physician diagnosis of diabetes. eGFR was calculated from standardized serum creatinine using the equation developed by the Chronic Kidney Disease Epidemiology Collaboration (CKD-EPI) [22]. Postmenopausal hormone use was determined based on inspection of medication bottles. Menopausal status was based on self-reported. Fasting serum insulin was measured using a radioimmunoassay (125Insulin Kit, Cambridge Medical Diagnostics, Billerica, Massachusetts) which was not specific for insulin and contained some cross-reactivity with pro-insulin. The details of the validity and reproducibility of this assay have been previously published [23].

In the HANDLS study, serum magnesium was measured using a spectrophotometry assay by Quest Diagnostics Nichols institute (Chantilly Virginia). Prevalent diabetes mellitus was defined as a having a fasting glucose level at least $126 \mathrm{mg} / \mathrm{dL}$ or self-reported of diabetes. eGFR was calculated from standardized serum creatinine using the CKD-EPI equation [22]. 


\section{Genotyping and imputation}

Details of genotyping, quality control, and imputation are reported in the Additional file 1: Supplementary Methods section. Briefly, genotyping was performed using the Affymetrix Genome-wide Human SNP Array 6.0 in the ARIC study and the Illumina $1 \mathrm{M}$ array in the HANDLS study. In both studies, individuals who were ancestry outlier or had cryptic relatedness were excluded. Imputation in AA participants in both studies was conducted using genotyped SNPs with MAF $>0.01$, call rate $>$ 0.95 , and Hardy-Weinberg equilibrium $p$-value $>0.00001$ and the 1000 Genomes March 2012 reference panels. The imputed genotype dosages were further filtered by MAF < 0.01 and imputation quality $<0.3$.

\section{Statistical methods for genome-wide association study and candidate Region interrogation}

We conducted a GWAS in the ARIC AA participants using linear regression with serum magnesium as the outcome controlling for age, center, gender, prevalent diabetes status, eGFR and its squared term, diuretic use, and genotype-based principal components with significant association $(p<0.05)$ with serum magnesium. The genetic model was assumed to be additive. Genome-wide significance was defined by a $\mathrm{p}$-value $<5 \times 10^{-8}$, and suggestive significance was defined by a $p$-value $<10^{-6}$. For the nine index SNPs for serum magnesium previously identified in EA populations, the significance threshold was set at 0.05 divided by the number of SNPs $(=0.006)$. Since allelic heterogeneity and differences in linkage disequilibrium pattern between EA and AA populations may give rise to different tag SNPs in an associated region, we evaluated the association of each locus previously identified in EA populations [6] in the GWAS results of ARIC AA participants based on a set of criteria similar to those developed in Liu et al. [24]. The region-specific significant threshold was defined as $\mathrm{p}$-value $<0.05$ divided by the number of independent SNPs in the region of the locus. The span of the region was defined as between the two closest recombination hot spots on both sides of the published index SNP, or the length of gene containing the index SNP, whichever was larger. The number of independent SNPs at each locus was determined using PLINK [25] by pruning SNPs with variance inflation factor $>2$ using a 50-SNP sliding window with a shift of 5 SNPs.

\section{Replication}

The index SNPs with suggestive significance in the GWAS of ARIC AA participants and the ARIC AA-specific index SNPs in known EA loci were sent forth for replication in the HANDLS study using the same regression model. The significant criteria for replication were set as $p$-value $<0.05$ with consistent direction of association. We performed fixed effect meta-analysis [26] combining the results from
AA participants of the ARIC and HANDLS studies. A known EA locus was considered to be associated in AA populations if the AA-specific index SNP from the ARIC study met the replication criteria and the region-specific significance threshold.

\section{Enhancer enrichment test}

Since loci associated with complex traits identified in GWAS tend to reside in regulatory regions [27], for the three AA-associated loci (MUC1, SHROOM3, and TRPM6) that were replicated in the HANDLS study, we conducted enhancer enrichment test using HaploReg version 2 [28]. At each locus, the SNPs used in the tests were those with $\mathrm{D}^{\prime}>0.9$ and $\mathrm{r}^{2}>0.2$ with the AA-specific index SNP based on 1000 Genomes Phase 1 AFR data and retrieved from HaploReg. The numbers of SNPs used were 58 at MUC1, 11 at SHROOM3, and 27 at TRPM6.

\section{Gene-environment interaction analyses for MUC1 and TRPM6}

To investigate gene-environment interaction, we focused on MUC1 and TRPM6. In addition to being the loci that had the largest effect estimates in EA participants, both loci are known to be involved in nutrient transport [10] with TRPM6 being a magnesium transporter [16]. Their gene functions support them as the causal genes in the GWAS-identified loci. The index SNP at MUC1 in EA populations, rs4072037, seems to be located in a regulatory region based on data from RegulomeDB [14] and in moderate linkage disequilibrium with an expression tag SNP of MUC1 (rs4971093 at 155,144,300 bp) based on data from the Genotype-Tissue Expression (GTEx) project [15]. We searched the literature for factors that influence the expression levels of MUC1 and TRPM6 and found that progesterone regulates $M U C 1$ [12], and insulin regulates TRPM6 [9]. Hence we tested for the effect modification between progestin use and $M U C 1$ and between fasting insulin levels and TRPM6 in relation to serum magnesium levels.

The analyses for gene-environment interaction were conducted within each self-reported race group in the following steps. First, the potential effect modifier was coded as a categorical variable. For progestin use, gender, postmenopausal status, and hormone use were combined into a categorical variable with 5 categories: males, premenopausal females, postmenopausal females with hormone therapy, postmenopausal females with non-progestin hormone therapy, and postmenopausal females with progestin use. Fasting insulin levels were categorized into two levels at $80 \mathrm{mmol} / \mathrm{L}$, which is considered as a threshold indicating insulin resistance $[29,30]$. Second, we tested for the independence between the most likely genotype of the index SNP and the potential effect modifier using Chisquare tests. Third, we performed linear regression using 
serum magnesium levels as the outcome, included the same covariates used in the GWAS of ARIC AA participants and tested for statistical interaction between the potential effect modifier and the index SNPs at the locus. Fourth, we evaluated the association between the index SNP and serum magnesium within each category of the effect modifier. For the evaluation of effect modification between insulin and the TRPM6 index SNP, participants who did not fast for 8 hours before blood drawn (182 in EA and 187 in AA) or were taking insulin (55 in EA and 61 in AA) were excluded, resulting in a sample size of 8689 in EA participants and 2488 in AA participants.

In addition, two nonsynonymous SNPs (nsSNPs) at TRPM6 were reported to inhibit the expression of TRPM6 in response to higher insulin levels (rs2274924 [K1584E] and rs3750425 [V1393I]) [9]. To determine whether the association between the GWAS index SNPs and serum magnesium were independent of these two nsSNPs, we estimated the linkage disequilibrium as measured by D' between the GWAS index SNPs and these two nsSNPs at TRPM6. D' was used as the measure of linkage disequilibrium here because it estimates whether two variants are on the same haplotype across allele frequencies. Next, we evaluated the association between these two nsSNPs and serum magnesium by fasting insulin levels with and without controlling for the GWAS index SNP at TRPM6.

To assess the required sample size for replicating the gene-environment interaction in other cohorts, we conducted a post-hoc power analysis by simulating the distributions of genotypes and effect sizes found in ARIC EA participants. Using an alpha of 0.05 , the power for detecting an interaction between the index SNP at MUC1 and postmenopausal females with progestin use versus male was $27 \%$ for a sample size of 3000, and $68 \%$ for a sample size of 9000 . For the interaction between the index SNP at TRPM6 and insulin levels, the power was $34 \%$ for a sample size of 3000, and $75 \%$ for a sample size of 9000 .

\section{Software}

Linkage disequilibrium in ARIC study as measured by $\mathrm{r}^{2}$ and D' was estimated using PLINK 1.07 [25] based on most likely genotypes derived from imputed dosage. The percentage of phenotypic variance explained by a SNP was estimated by calculating the effect size of the SNP $\left(2 \beta^{2} x M A F x[1-M A F]\right)[31]$ divided by the phenotypic variance. Regression analyses were performed using the imputed dosage of the genotype. GWAS was conducted using SNPTEST 2.4.1 [32]. Fixed effect meta-analyses were conducted using metal [26]. Other analyses were performed using R. The present study has been approved by the respective Institutional Review Boards of the ARIC study and the HANDLS study and was performed in accordance with the Declaration of Helsinki. All participants provided informed consent.

\section{Availability of supporting data}

The data set supporting the results of this article is available in the $\mathrm{dbGaP}$ repository, phs000090.v2.p1

(http://www.ncbi.nlm.nih.gov/projects/gap/cgi-bin/study. cgi?study_id=phs000090.v2.p1).

\section{Additional files}

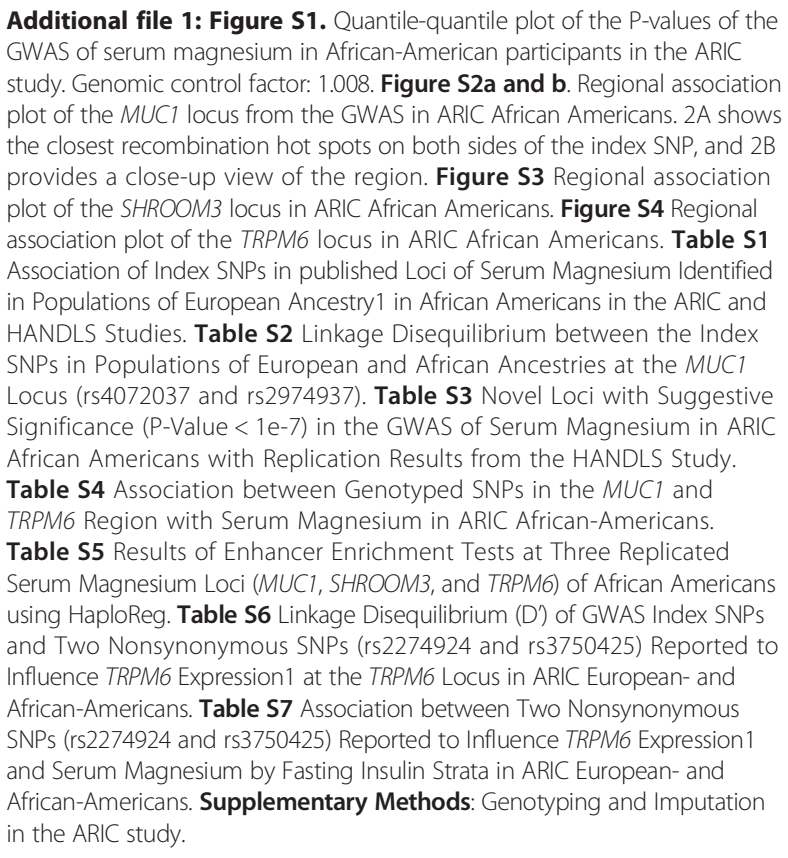

\section{Competing interests}

The authors declare that they have no competing interests.

\section{Authors' contributions}

$A T, A K, A F$, and WK contributed to the design of the present study. AT, ST, and $M N$ conducted the statistical analyses. AK, AF, NM, MN, ME, AZ, CF, EB, $J C$, and WK drafted the manuscript. All authors read and approved the final manuscript.

\section{Acknowledgements}

The authors thank the staff and participants of the ARIC study for their important contributions.

\section{Funding}

Dr. Tin is supported by the NIH/NIDDK T32 DK007732 Renal Disease Epidemiology Training Program.

The Atherosclerosis Risk in Communities Study is carried out as a collaborative study supported by National Heart, Lung, and Blood Institute contracts (HHSN268201100005C, HHSN268201100006C,

HHSN268201100007C, HHSN268201100008C, HHSN268201100009C, HHSN268201100010C, HHSN268201100011C, and HHSN268201100012C). AK was supported by the Emmy Noether Programme of the German Research Foundation (KO 3598/2-1).

\section{Author details}

'Johns Hopkins Bloomberg School of Public Health, Baltimore, MD, USA. ${ }^{2}$ University Medical Center Freiburg, Freiburg, Germany. ${ }^{3}$ University of Minnesota School of Public Health, Minneapolis, MN, USA. ${ }^{4}$ Johns Hopkins University School of Medicine, Baltimore, MD, USA. ${ }^{5}$ National Institute on Aging, National Institutes of Health, Bethesda, MD, USA. ${ }^{6}$ University of Mississippi Medical Center, Jackson, MS, USA. 7University of Texas School of Public Health, Houston, TX, USA. 
Received: 6 August 2014 Accepted: 15 May 2015

Published online: 29 May 2015

\section{References}

1. Del Gobbo LC, Imamura F, Wu JH, de Oliveira Otto MC, Chiuve SE, Mozaffarian D. Circulating and dietary magnesium and risk of cardiovascular disease: a systematic review and meta-analysis of prospective studies. Am J Clin Nutr. 2013;98(1):160-73.

2. Peacock JM, Folsom AR, Arnett DK, Eckfeldt JH, Szklo M. Relationship of serum and dietary magnesium to incident hypertension: the Atherosclerosis Risk in Communities (ARIC) Study. Ann Epidemiol. 1999;9(3):159-65.

3. Kao WH, Folsom AR, Nieto FJ, Mo JP, Watson RL, Brancati FLCINAIMO, et al. Serum and dietary magnesium and the risk for type 2 diabetes mellitus: the Atherosclerosis risk in communities study. Arch Intern Med. 1999;159(18):2151-9.

4. Tin A, Grams ME, Maruthur NM, Astor BC, Couper D, Mosley TH, et al. Coresh J. Results from the Atherosclerosis Risk in Communities study suggest that low serum magnesium is associated with incident kidney disease. Kidney Int: Kao WH; 2014.

5. Hunter DJ, Lange M, Snieder H, MacGregor AJ, Swaminathan R, Thakker RV, et al. Genetic contribution to renal function and electrolyte balance: a twin study. Clin Sci (Lond). 2002;103(3):259-65.

6. Meyer TE, Verwoert GC, Hwang SJ, Glazer NL, Smith AV, van Rooij FJ, Ehret GB, Boerwinkle E, Felix JF, Leak TS et al:: Genome-wide association studies of serum magnesium, potassium, and sodium concentrations identify six Loci influencing serum magnesium levels. PLoS Genet 2010, 6(8).

7. Resnick LM, Bardicef O, Altura BT, Alderman MH, Altura BM. Serum ionized magnesium: relation to blood pressure and racial factors. Am J Hypertens. 1997;10(12 Pt 1):1420-4.

8. Frazer KA, Ballinger DG, Cox DR, Hinds DA, Stuve LL, Gibbs RA, et al. A second generation human haplotype map of over 3.1 million SNPs. Nature. 2007:449(7164):851-61.

9. Nair AV, Hocher B, Verkaart S, van Zeeland F, Pfab T, Slowinski T, et al. Loss of insulin-induced activation of TRPM6 magnesium channels results in impaired glucose tolerance during pregnancy. Proc Natl Acad Sci U S A. 2012;109(28):11324-9.

10. McAuley JL, Linden SK, Png CW, King RM, Pennington HL, Gendler SJ, et al. MUC1 cell surface mucin is a critical element of the mucosal barrier to infection. J Clin Invest. 2007;117(8):2313-24.

11. Leroy X, Copin MC, Devisme L, Buisine MP, Aubert JP, Gosselin B, et al. Expression of human mucin genes in normal kidney and renal cell carcinoma. Histopathology. 2002;40(5):450-7.

12. Meseguer M, Aplin JD, Caballero-Campo P, O'Connor JE, Martin JC, Remohi $J$, et al. Human endometrial mucin MUC1 is up-regulated by progesterone and down-regulated in vitro by the human blastocyst. Biol Reprod. 2001;64(2):590-601.

13. Horne AW, Lalani EN, Margara RA, White JO. The effects of sex steroid hormones and interleukin-1-beta on MUC1 expression in endometrial epithelial cell lines. Reproduction (Cambridge, England). 2006;131(4):733-42.

14. Boyle AP, Hong EL, Hariharan M, Cheng Y, Schaub MA, Kasowski M, et al. Annotation of functional variation in personal genomes using RegulomeDB. Genome Res. 2012;22(9):1790-7.

15. GTEx. The Genotype-Tissue Expression (GTEx) project. Nat Genet. 2013:45(6):580-5.

16. Schlingmann KP, Gudermann T. A critical role of TRPM channel-kinase for human magnesium transport. J Physiol. 2005;566(Pt 2):301-8.

17. de Baaij JH, Groot Koerkamp MJ, Lavrijsen M, van Zeeland F, Meijer H, Holstege FC, et al. Elucidation of the distal convoluted tubule transcriptome identifies new candidate genes involved in renal Mg2+ handling. Am J Physiol Renal Physiol. 2013;305(11):F1563-73.

18. ARIC. The Atherosclerosis Risk in Communities (ARIC) Study: design and objectives. The ARIC investigators. Am J Epidemiol. 1989;129(4):687-702.

19. Coburn JW, Popovtzer MM, Massry SG, Kleeman CR. The physicochemical state and renal handling of divalent ions in chronic renal failure. Arch Intern Med. 1969;124(3):302-11.

20. Evans MK, Lepkowski JM, Powe NR, LaVeist T, Kuczmarski MF, Zonderman AB. Healthy aging in neighborhoods of diversity across the life span (HANDLS): overcoming barriers to implementing a longitudinal, epidemiologic, urban study of health, race, and socioeconomic status. Ethn Dis. 2010;20(3):267-75.
21. Gindler EM, Heth DA. Colorimetric determination with bound "calmagite" of magnesium in human blood serum (abstract). Clin Chem. 1971;17:662.

22. Levey AS, Stevens LA, Schmid CH, Zhang YL, Castro 3rd AF, Feldman HI, et al. A new equation to estimate glomerular filtration rate. Ann Intern Med. 2009;150(9):604-12.

23. Eckfeldt JH, Chambless LE, Shen YL. Short-term, within-person variability in clinical chemistry test results experience from the Atherosclerosis risk in communities study. Arch Pathol Lab Med. 1994;118(5):496-500.

24. Liu CT, Garnaas MK, Tin A, Kottgen A, Franceschini N, Peralta CA, et al. Genetic association for renal traits among participants of African ancestry reveals new loci for renal function. PLoS Genet. 2011;7(9), e1002264.

25. Purcell S, Neale B, Todd-Brown K, Thomas L, Ferreira MA, Bender D, et al. PLINK: a tool set for whole-genome association and population-based linkage analyses. Am J Hum Genet. 2007;81(3):559-75.

26. Willer CJ, Li Y, Abecasis GR. METAL: fast and efficient meta-analysis of genomewide association scans. Bioinformatics. 2010;26(17):2190-1.

27. Maurano MT, Humbert R, Rynes E, Thurman RE, Haugen E, Wang H, et al. Systematic localization of common disease-associated variation in regulatory DNA. Science. 2012;337(6099):1190-5.

28. Ward LD, Kellis M. HaploReg: a resource for exploring chromatin states, conservation, and regulatory motif alterations within sets of genetically linked variants. Nucleic Acids Res. 2012;40(Database issue):D930-4.

29. Ascaso JF, Pardo S, Real JT, Lorente RI, Priego A, Carmena R. Diagnosing insulin resistance by simple quantitative methods in subjects with normal glucose metabolism. Diabetes Care. 2003;26(12):3320-5.

30. McAuley KA, Williams SM, Mann JI, Walker RJ, Lewis-Barned NJ, Temple LA, et al. Diagnosing insulin resistance in the general population. Diabetes Care. 2001;24(3):460-4.

31. Park JH, Wacholder S, Gail MH, Peters U, Jacobs KB, Chanock SJ, et al. Estimation of effect size distribution from genome-wide association studies and implications for future discoveries. Nat Genet. 2010;42(7):570-5.

32. Marchini J, Howie B. Genotype imputation for genome-wide association studies. Nat Rev Genet. 2010;11(7):499-511.

\section{Submit your next manuscript to BioMed Central and take full advantage of:}

- Convenient online submission

- Thorough peer review

- No space constraints or color figure charges

- Immediate publication on acceptance

- Inclusion in PubMed, CAS, Scopus and Google Scholar

- Research which is freely available for redistribution 